\title{
Entre la convención y el futuro: la traducción en la época de la diferencia
}


No resulta descabellado afirmar que hoy, en un mundo en el que la publicidad lo permea todo, tal vez hasta la evolución epistemológica de las disciplinas académicas se produzca en buena medida a golpe de sonoros logos y pegadizos reclamos. En nuestro ámbito, sin ir más lejos, algunos de los cambios más significativos que últimamente ha experimentado la doxa disciplinaria deben mucho a fórmulas tan vibrantes como las que corean que «toda traducción implica una manipulación» o que «traducir es un acto político». Ciertamente, el hecho de que, mediante la repetición del logo, se haya logrado la generalización y la aceptación unánime de unas concepciones, en principio, tan polémicas refleja la efectividad, y hasta la necesidad, de que a las teorías se les administren periódicamente inyecciones con nuevos tropos para que puedan seguir pensando más allá de los esquemas convencionales y, por ende, para evitar que la disciplina y la profesión se anquilosen en los modelos establecidos, con los ojos cerrados a los retos que le plantea la sociedad en la que operan. Con todo, en virtud de la misma dinámica, también es preciso estar alerta y saber hurtarse a la tentación simplificadora que acompaña a estos resultones asertos, y tomarlos como adalides de la novedad y no como la novedad en sí misma. Entre otras cosas, porque la novedad científica muchas veces no radica en cambiar drásticamente la visión de las cosas y tirar por la borda el conocimiento acumulado hasta la fecha, sino en completarlo o perfeccionarlo de manera original y relevante.

En este sentido, en las últimas décadas la traductología se ha regocijado en reiterar que la traducción no es meramente (o incluso sin el «meramente») una operación lingüística, sino una mediación cultural. Y, a pesar de lo incuestionable de la frase (por lo menos, en la actualidad), tal vez haya llegado el momento de cuestionar, o matizar, algunas de sus lecturas. Y es que, aunque al recordarlo resulte evidente, esa «intermediación cultural» no se restringe a la negociación de lo que en otro tiempo se denominó culturemas, y mucho menos únicamente en virtud de la afamada pero restrictiva dicotomía venutina de la extranjerización vs. domesticación. «Cultura», en efecto, son prototípicamente los rasgos y productos típicos de una sociedad, las referencias locales, y los usos y costumbres, pero también, y de hecho como integrantes de pleno derecho de esa última categoría, los usos y costumbres lingüísticas y textuales; esto es, las rutinas convencionalizadas que con fuerza magnética penden sobre los emisores a la hora de escribir textos. De ahí que, aunque en su momento la frase emblema del «culturalismo» resultara muy beneficiosa para ampliar los horizontes de la traductología más allá de los intereses contemporáneos de la lingüística, no cabe entender estas aproximaciones a la traducción, como reza otra afirmación que va camino de convertirse en lugar común en nuestra rama, como enfoques enfrentados, sino más bien como complementarios. Y, lo cierto es que, a pesar del grado de reiteración de que a estas alturas ya goza el matiz, aún cabe calificarlo de declaración de intenciones, por cuanto son escasas las apuestas investigadoras que asumen materializar el reto. Una de las excepciones a lo dicho se encuentra en el cuarto libro de la serie Traducción y cultura (Málaga, Encasa, 2006), editado por Leandro Félix Fernández y Carmen Mata Pastor, que, centrado en cuestiones de «Convenciones textuales y estrategia traslativa», explota esta conjunción disciplinaria, como recuerdan los editores, sumamente reveladora tanto para el ejercicio profesional de la traducción como para su didáctica. No en vano, aunque el traductor medie entre culturas, los encargos que dan pie al ejercicio de su labor se 
concretan en textos; textos con un pasado intertextual y, por tanto, portadores de una cohorte de expectativas que condicionan la aceptabilidad de la versión traducida. El libro, además de ofrecer interesantes reflexiones en el plano teórico sobre las implicaciones de tal circunstancia, aporta numerosos consejos prácticos de suma utilidad en los diferentes ámbitos profesionales en los que bucean sus colaboradores: jurídicoadministrativo, publicitario y turístico, literario, periodístico, etc.

Así, ya en el primero de los artículos de esta antología, Hannelore Benz Busch alerta de la importancia que reviste observar, cara a la traducción de determinados géneros, las convenciones vigentes en la cultura de llegada, que, en palabras de esta autora, «son la tarjeta de visita según la cual nos juzgan, al menos en una primera instancia». A través de un repaso contrastivo por una serie de problemas patentes en ciertos tipos textuales representativos, como la correspondencia privada y comercial, el certificado de trabajo o los textos periodísticos, se ilustra a qué tipo de malentendidos culturales puede abocar una traducción mecánica que minimice la importancia de la dispar percepción con que se reciben los patrones retóricos convencionalizados en las diferentes culturas implicadas en el trasvase, y se resalta la necesidad de hacer hincapié en esta dimensión en la formación de traductores.

A similares conclusiones llega en un artículo situado en el ámbito jurídico José Antonio Gallegos Rosillo, quien, centrándose en los escollos particulares que plantea al traductor el desfase actual entre los dictados de la gramática normativa y el uso en cuanto al empleo de las formas verbales relativas a la expresión de la obligación en los textos legislativos franceses, destaca la importancia de someter a los textos que han de traducirse a un análisis macro y microcontextual que desvele el valor pragmático que encubren las formulaciones ritualizadas, de cuya especificidad en ocasiones ni siquiera dan cuenta las obras de referencia y autoridad a las que acuden los traductores buscando respuestas a sus incertidumbres.

En el ámbito de estudio y en las ventajas de realizar un análisis a fondo coincide también la Dra. Valderrey Reñones, de la Universidad de Salamanca, quien precisamente en el siguiente artículo presenta un modelo abarcador con el que adentrarse en los distintos niveles de los textos jurídicos (comunicativo, pragmático, semántico-cognitivo y formal) con vistas a deducir el funcionamiento de este tipo de documentos, donde tanta trascendencia tienen, tanto en el plano del significado como de la credibilidad y la autoridad textual, la ritualización retórica y la regularidad. De hecho, desde este convencimiento de base, la autora se adscribe, tras presentar una revisión crítica y muy informada de los distintos enfoques que se han preocupado de la caracterización de los lenguajes de especialidad, a las últimas aproximaciones de tipo textual y discursivo que explotan la utilidad analítica y didáctica del concepto de género. Este rigurosísimo trabajo ofrece una sólida fundamentación teórica para que el traductor o el aprendiz, provisto de una metodología de análisis y de trabajo, se aventure a transgredir la norma por defecto de la versión literal para poner en práctica soluciones de traducción sofisticadas que le reporten credibilidad discursiva ante la comunidad de expertos para la que trabaja.

Además de una negociación retórica entre rutinas del discurso divergentes, la traducción exige y/o implica asimismo un ajuste de las percepciones intersubjetivas en torno al Otro; esto es, el acomodo de elementos de la cultura de origen en otra sociedad donde tiene hecha una 
imagen, una reputación más o menos idealizada, una serie de ideas preconcebidas que lo condicionan. El ámbito de la publicidad constituye un lugar idóneo para comprobar la vigencia, y la importancia, de estos estereotipos culturales, y en él se adentra la Dra. Marie-Ange Bugnot. Así, en su artículo presenta en primer lugar una caracterización de los textos turísticos, especialmente en lo referente a las funciones ulteriores que cumplen y los esquemas retóricos que emplean para conseguirlas. A partir de ahí, reflexiona sobre los problemas que surgen en la traducción de estos textos. Como no podía ser de otra manera, la autora repara en los peajes impuestos por la intertextualidad que hereda el texto original cuando trata de hacerse un hueco en la cultura de llegada y en los percances que, por mor de la existencia de clichés sobre el Otro, pueden sufrir los elementos transportados en el periplo transcultural. Como se comprueba con el análisis concreto de una guía turística publicada en español y francés, que suscita lecturas muy distintas en cada caso, la traducción en el sector turístico es una operación delicada cuyo éxito requiere un verdadero ejercicio de diplomacia intercultural.

De hecho, así lo saben los responsables de las campañas de promoción turística internacionales, en las que bucea el siguiente capítulo. La Dra. Cómitre explica, tomando como ejemplo el caso concreto de "España marca», los criterios manejados en el proceso de creación y selección de una campaña publicitaria, así como los filtros y cribas por las que pasa para su posterior difusión en otras culturas. La complejidad que entraña la labor de los profesionales al cargo se ilustra con el análisis concreto de los indicadores de convenciones estilísticas culturales y, más específicamente, de las estrategias puestas en marcha para mantener su efectividad en la cultura de llegada. La lectura de este artículo pone

de manifiesto la importancia de saber conjugar el skopos de la traducción con el horizonte de expectativas de las que parte el lector meta; una importancia que, en último extremo, en el sector turístico se calibra en términos económicos. Y, ciertamente, no es una excepción: el artículo siguiente lo demuestra a las claras.

Efectivamente, el capítulo a cargo de Inmaculada Serón Ordóñez, centrado en los informes corporativos, se inicia precisamente con esa reflexión, pues la traducción de este género textual redunda en la ampliación o la reducción de las oportunidades de negocio de las compañías transnacionales. La autora, a través de la comparación y análisis de la macroestructura y los patrones de distribución de la información de un corpus compuesto por balances de situación de informes anuales originalmente redactados en español, inglés y francés, y por otros traducidos al inglés ilustra las dificultades que acompañan a la traducción de un tipo de texto que presenta gran disparidad cultural en cuanto a convenciones textuales pero que por otra parte está marcadamente formalizado. Además, recuerda cómo a los problemas derivados de conciliar estos estilos divergentes se añaden otros factores de diferente índole, si se quiere, político-estratégicos, como la conveniencia de atender en la traducción a las restricciones que imponen, por ejemplo en el plano de la terminología, las normas internacionales con que se trata de conciliar la diversidad de prácticas contables. De la lectura de este informado estudio se deduce, en primer lugar, la complejidad de la labor del traductor especializado, en este caso en el área de las finanzas, que nunca se restringe a la concatenación mecánica de los equivalentes terminológicos de los elementos del texto fuente; en segundo lugar, el grado de conocimiento sobre el ámbito particular que exige el ejercicio de la traducción especializada; y, por último, el 
alcance de la responsabilidad que carga el traductor a sus espaldas, es decir, la trascendencia real de sus actos, que, en este caso, puede incluso calcularse numéricamente.

Aunque en otros ámbitos esa trascendencia es difícilmente cuantificable, no es por ello menos importante, en tanto entronca de manera directa con la dimensión ética. Los siguientes artículos del libro lo tienen presente y lo ponen de relieve de distintas maneras.

El que corre a cargo del Dr. Leandro Félix, que entre otras cosas recoge una experiencia didáctica en la que la concienciación de los alumnos sobre los usos y valores de los marcadores de la modalidad subjetiva y objetiva reporta beneficios a las traducciones resultantes, alerta de la necesidad de aprender a leer y a transmitir correctamente la intencionalidad que subyace a todo texto. Saber desentrañar el valor pragmático de las decisiones que el autor toma, los elementos que elige de una gama siempre limitada por las convenciones vigentes, es un paso vital para trasladar su programa conceptual con eficacia en otra cultura.

Esa eficacia traslativa a menudo pasa por la gestión conveniente de los abismos culturales, por la introducción de explicaciones culturales sin las cuales el texto resultaría abstruso o impenetrable. María José Hernández Guerrero estudia en su colaboración al volumen las técnicas más habituales para introducir información añadida en el ámbito periodístico. Así, el análisis de un corpus le permite detectar las convenciones seguidas en los textos traducidos, que en algunos casos divergen de las observadas en los textos concebidos originalmente en español. Se trata, en definitiva, de ver en qué medida la traducción cumple en este aspecto los parámetros de aceptabilidad establecidos o, a la inversa, hasta qué punto goza de licencia para incumplirlos. En este trabajo se percibe que las decisiones del traductor nunca son neutrales, por mínimo que en principio pueda parecer su calado: así, la utilización de una u otra estrategia ortotipográfica para añadir información encubre importantes cuestiones ideológicas, como la visibilización o el ocultamiento de la polifonía que está presente en toda traducción o el éxito o el fracaso de la mediación intercultural.

Las cuestiones ideológicas también destacan en el artículo de Guadalupe Ruiz Yepes, quien recuerda la importancia que reviste para el traductor conocer las normas de escritura de las culturas de origen, en la medida en que los autores transmiten aspectos pragmáticos e ideológicos bien acogiéndose a las convenciones estilísticas bien violentándolas. $\mathrm{El}$ conocimiento de la regularidad, pues, se descubre fundamental para avistar las desviaciones que obedecen a otros factores pragmáticos o los desvelan.

En este sentido, cabe extraer la conclusión de que los textos siempre cobran (nuevo) significado a la luz de otros, en comparación con el intertexto con el que se relacionan.

La cuestión de la intertextualidad recibe notable atención en el capítulo de Estefanía Flores Acuña, que examina las traducciones al español de uno de los cómics italianos más conocidos en nuestro país: Lupo Alberto. Consciente de la influencia de numerosos factores interrelacionados en la recepción de la traducción, el estudio aborda diversos niveles: desde las cuestiones macroculturales (como la explicación en torno a la posición que ocupa el cómic italiano, y concretamente Lupo Alberto, en el polisistema español), pasando por las características del texto origen, hasta llegar al tratamiento microtextual que reciben en la traducción al castellano ciertos elementos (por ejemplo, el nivel de lengua, las interjecciones, los marcadores discursivos o los elementos intertextuales). 
Un estudio comparativo similar, si bien centrado en un aspecto mucho más concreto, ocupa parte del artículo de la Dra. Carmen Mata Pastor, quien a través de los escollos que plantea un elemento en apariencia superficial, ortotipográfico, como la cursiva, recuerda, tal vez como llamada de atención frente a la tiranía de la literalidad acrítica, la multiplicidad de estrategias que tiene a su disposición el traductor ante elementos cuyos usos y valores se demuestran asimétricos en las diferentes culturas. Además de aportar un sólido aparataje teórico para entender las dificultades de traducción de la cuestión específica que aborda y de ofrecer una interesante selección de las tan osadas como acertadas soluciones de los traductores de Roald Dahl al castellano y al italiano, este artículo reaviva la necesidad de dudar que es consustancial al ejercicio de la profesión. Efectivamente, puesto que la diversidad cultural anida incluso en lo que una primera mirada pudiera juzgar accesorio, la traducción exige una constante reflexión sobre los límites de la pleitesía a la Otredad y las restricciones que imponen el uso y la convencionalización textual. La traducción obliga siempre a pensar hasta la extenuación en la diferencia.

Y precisamente el ánimo de concienciar sobre la diferencia y su pluralidad constitutiva preside el último artículo de esta interesante antología, a cargo de M. ${ }^{a}$ Gracia Torres Díaz e Iqbal Bains, quienes exponen la razón de ser y los contenidos de la herramienta didáctica Interpreta. Se trata éste de un DVD concebido

para la práctica de la interpretación, editado por la Universidad de Málaga, que, frente a los materiales tradicionales, incorpora múltiples variedades de inglés y contenidos temáticos en sintonía con la era multicultural en la que habitamos. Además de proponerse ensanchar los conocimientos de los estudiantes y afinar su sensibilidad hacia la diversidad, los prepara para atender los retos de la traducción en una sociedad, la nuestra, que a pasos agigantados cambia su composición y que, por tanto, también a pasos agigantados exige de las profesiones que pongan a prueba su capacidad evolutiva y que renueven su compromiso con la sociedad en la que operan; en definitiva, que asuman su responsabilidad.

En este sentido, cabe afirmar que, como denominador común, todos los trabajos que conforman el cuarto volumen de Traducción y cultura animan a seguir pensando en los desafíos que se abren para la traducción en el mañana. Desde un punto de partida por otra parte aún necesitado de investigación como es el de las convenciones textuales, esta obra, además de dar una serie de respuestas útiles y concretas, anima al traductor profesional o en ciernes a seguir haciéndose preguntas. $Y$ es que, en la medida en que traducir es mediar entre diferencias, en una época en la que éstas se agrandan y en la que, por otra parte, hemos asumido el compromiso de respetarlas, lejos de regocijarnos en los modelos heredados, necesitamos seguir ideando nuevas fórmulas. 